\title{
Finite-temperature phase diagram of (111) nickelate bilayers
}

\author{
Oleg Janson* and Karsten Held \\ Institut für Festkörperphysik, TU Wien, Wiedner Hauptstraße 8-10, 1040 Vienna, Austria
}

(Dated: September 20, 2019)

\begin{abstract}
We report a density functional theory plus dynamical mean field theory (DFT+DMFT) study of an oxide heterostructure of $\mathrm{LaNiO}_{3}$ (LNO) bilayers in [111] direction interleaved with four atomic monolayers of $\mathrm{LaAlO}_{3}$. DFT $+U$ optimizations yield two stable solutions: a uniform structure with equivalent $\mathrm{NiO}_{6}$ octahedra, as well as a bond-disproportionated (BD) structure featuring a breathing distortion. For both structures, we construct the low-energy models describing the Ni $e_{g}$ states by means of Wannier projections supplemented by the Kanamori interaction, and solve them by DMFT. Using the continuous-time quantum Monte Carlo algorithm in the hybridization expansion, we study the temperature range between 145 and $450 \mathrm{~K}$. For the uniform and the BD structure, we find similar phase diagrams that comprise four phases: a ferromagnetic metal (FM), a paramagnetic metal (PM), an antiferro-orbitally-ordered insulator (AOI), as well as a paramagnetic insulator (PI). By calculating momentum-resolved spectral functions on a torus and a cylinder, we demonstrate that the FM phase is not a Dirac metal, while both insulating phases are topologically trivial. By a comparison with available experimental data and model DMFT studies for the two-orbital Hubbard model, we suggest that LNO bilayers are in the AOI phase at room temperature.
\end{abstract}

\section{INTRODUCTION}

Transition metal oxides (TMO) exhibit a plethora of fascinating physical behaviors, such as metal-insulator transitions [1], multiferroicity [2-4], colossal magnetoresistance $[5,6]$, and high-temperature superconductivity $[7,8]$. The ongoing progress in fabricating highquality TMO thin films led to the emergence of a new class of artificial materials: oxide heterostructures. Their properties are often markedly different from their TMO constituents. An archetypical example are heterostructures of two band insulators, $\mathrm{SrTiO}_{3}$ (STO) and $\mathrm{LaAlO}_{3}$ (LAO): if the latter component reaches the critical thickness of four atomic monolayers, a two-dimensional (2D) electron gas emerges at the interface [9]. Even more striking effects are observed for TMO with a partly filled $d$ shell. Here, electronic correlations become essential. For example, the emergence of a ferromagnetic metal at $\mathrm{LaMnO}_{3} / \mathrm{SrMnO}_{3}$ interfaces was reported [10], where both constituents are bulk antiferromagnetic insulators; or the polar field and the Mott insulating gap of $\mathrm{STO} / \mathrm{LaVO}_{3}$ can be employed as a solar cell $[11,12]$.

Although the variety of TMO gives rise to a very large number of possible binary combinations, the count of studied oxide heterostructures grows slowly. Following the pioneering works of Hwang and Ohtomo [9, 13], the research has been largely focused on superlattices whose constituents have a (possibly distorted) perovskite structure in the bulk, and the direction of growth was typically chosen to be along the (pseudo)cubic [001] direction. Against this backdrop, Xiao et al. [14] argued that bilayers grown along the trigonal axis, i.e. in the [111] direction, form a honeycomb lattice, with an excellent potential to create correlated analogs of graphene [14]. By employing the tight-binding (TB) approximation, they

\footnotetext{
*olegjanson@gmail.com
}
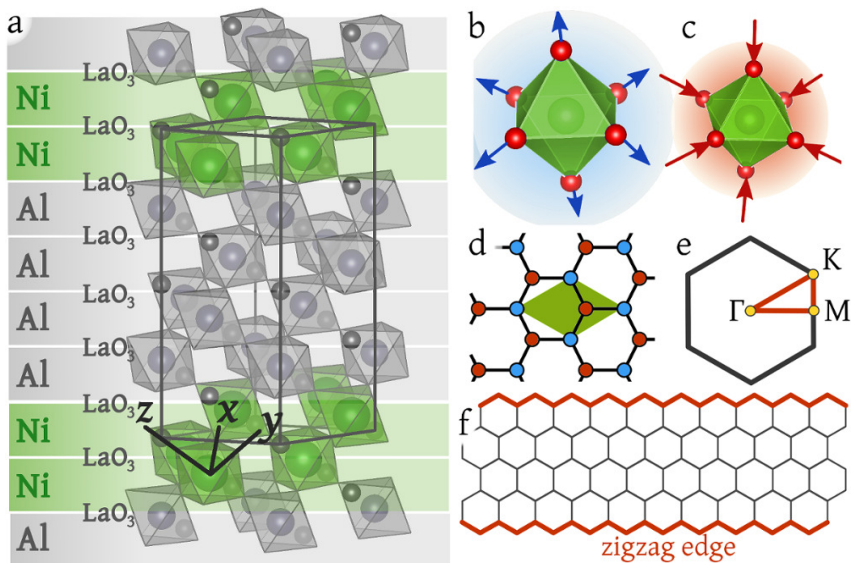

FIG. 1. (Color online) (a) In the trigonal unit cell used for DFT+DMFT calculations, (111) nickelate bilayers are separated by four $\mathrm{LaAlO}_{3}$ layers. For $\mathrm{NiO}_{6}$ octahedra, the local coordinate axes $x, y, z$ are indicated. Bond disproportionation gives rise to a breathing distortion: stretched (b) and squeezed (c) $\mathrm{NiO}_{6}$ octahedra alternate in the lattice (d). (e) High-symmetry points of the Brillouin zone (BZ). (f) Zigzag edges of the honeycomb lattice used for the edge state calculations.

studied different fillings of the correlated $d$ shell in the presence of the spin-orbit coupling (SOC), and demonstrated that such (111) bilayers can host various topologically nontrivial phases. Also Haldane's [15] quantum anomalous Hall state can be realized in (111) bilayers of $\mathrm{SrRuO}_{3}$ on STO [16].

The ensuing numerical studies extended the TB analysis by including electronic interaction effects on a meanfield level $[17,18]$, and identified $\mathrm{LaNiO}_{3}$ bilayers (2LNO) in an LAO matrix as a promising candidate for the realization of topological states $[17,19]$. In the simplest ionic approximation, $\mathrm{Ni}^{3+}$ has the $d^{7}$ electronic configuration, whereby six electrons fully occupy the low-lying $t_{2 g}$ states 
and render them inactive. Hence, all change, orbital, and spin degrees of freedom in the $2 \mathrm{LNO} / n \mathrm{LAO}$ superlattices (Fig. 1, a) pertain to the single electron in the $e_{g}$ orbitals that remain degenerate in the trigonal symmetry [14]. This resilient degeneracy, in contrast to (001) superlattices [20], gives room for spontaneous ordering of complex orbitals and thus topologically nontrivial states emerge despite the small SOC [18]. While first analyses involving realistic tight-binding Hamiltonians evaluated by means of density functional theory (DFT) [19] as well as $\mathrm{DFT}+U$ calculations $[19,21]$ suggested the stability of a Dirac semimetal state, later $\mathrm{DFT}+U$ studies established a key role of a breathing distortion of $\mathrm{NiO}_{6}$ octahedra (Fig. 1 b, c) which opens a gap and competes with the topological states [21,22]. The breathing distortion is accompanied by a polarization, rendering (111) LNO bilayers a prospective multiferroic with a sizable spin polarization [22].

On the experimental side, transport measurements indicated a semiconducting behavior, with the gap showing a sizable dependence on the thickness of the LAO layer [23]. Recently, both the activated behavior and the sensitivity of the gap size were corroborated by an independent study, which reported gaps between 17 and $162 \mathrm{meV}$ for different LAO thicknesses [24]. The nature of the gap, in particular whether it is topological or related to a breathing distortion, remains an open question.

Here, we employ a combination of DFT and dynamical mean-field theory (DMFT) [25-29] to explore the phase diagram of 2LNO/4LAO heterostructures (Fig. 1, a). By performing detailed structural relaxations, we demonstrate that the presence of a breathing distortion can be neither proved, nor disproved: both structure types feature the same $\mathrm{DFT}+U$ total energies within the error bars. Hence, we carry out DFT+DMFT calculations for the uniform as well as the bond-disproportionated structure. Both show, despite the symmetry breaking associated with the distortion, actually quite similar physics.

This paper is organized as follows. The methods employed, including the structure optimization, DMFT, and the evaluation of spectral functions, are described in Sec. II. DFT+DMFT results for the uniform and the bond-disproportionated structures are presented in Sec. III. A discussion of the topological properties as well as comparison of our numerical results with the available experimental data are given in Sec. IV. We conclude our paper and provide a brief outlook in Sec. V.

\section{METHOD}

\section{A. Optimization of the crystal structure}

DFT + DMFT results generally depend on the structural input from DFT calculations. Hence, accurate information on the crystal structure is of crucial importance. This is particularly challenging for superlattices that are not amenable to standard x-ray or neu- tron diffraction measurements. A common approach is to evaluate the structural input computationally, by allowing for a relaxation of the atomic coordinates, but keeping the lattice constants fixed to that of the substrate and minimizing the total energy [29]. In contrast, for correlated materials, the underestimation of electronic correlations can have drastic impact on the crystal structure. A prominent example is $\mathrm{KCuF}_{3}$, where orbital ordering can give rise to a distortion of the lattice, known as the cooperative Jahn-Teller effect. This can be assisted by lattice effects, which may also be a driving force. While conventional DFT functionals yield a spurious undistorted structure, $\mathrm{DFT}+U$ captures the underlying physics and reproduce the experimentally observed distortion [30].

A distinct trait of bulk nickelates is their tendency towards bond disproportionation, i.e. developing a breathing distortion of $\mathrm{NiO}_{6}$ octahedra (e.g., [31-33]). Although it is not the case for bulk $\mathrm{LaNiO}_{3}$, the compressive strain exerted by the $\mathrm{LaAlO}_{3}$ substrate can stabilize the respective distortion. Similarly to $\mathrm{KCuF}_{3}$, this physics is not captured by DFT, and conventional functionals disfavor such a bond disproportionation [21]. Hence, structural optimizations of nickelate superlattices performed using a conventional DFT functional can possibly lead to spurious results, and correlations have to be accounted for in the course of a structural optimization. The optimal solution would be a self-consistent DFT+DMFT scheme with an atomic force calculation at every step, but such calculations require enormous computational efforts and remain unfeasible for multisite and multi-orbital systems such as nickelate heterostructures. Therefore, in this work, we restrict ourselves to DFT $+U$ structural optimizations that generally capture the structural details in the rare earth nickelates [34].

We employ the generalized gradient approximation (GGA) $+U$ functional with $U$ in the range 4.0 to $6.0 \mathrm{eV}$ and $J=1.0 \mathrm{eV}$ as implemented in VASP-5.3 [35]. Ionic relaxations are performed until all forces are below $0.005 \mathrm{eV} / \AA$. The in-plane unit cell parameters are fixed to that of bulk LAO. To optimize the $c$ parameter, we construct cells with different $c$ and subsequently optimize the atomic coordinates. In this way, we find that $c=13.30 \AA$ yields the lowest total energy independent of the $U$ value. Next, we consider two trial structures as a starting point - a uniform structure and a structure with a breathing distortion (BD), see Fig. 1 b,c - and relax the atomic coordinates by keeping the unit cell parameters fixed. Despite the general trend that the uniform structure has a lower energy for smaller $U_{d}$, the energy differences are of the order of several $\mathrm{K}$ per cell, i.e. on a par with the accuracy of DFT total energies. We conclude that the elastic energy due to $\mathrm{BD}$ and the concomitant change in the electric potential are well-balanced, so that $\mathrm{DFT}+U$ calculations cannot provide an unambiguous answer, which of the two structure types is realized in $2 \mathrm{LNO} / 4 \mathrm{LAO}$. We therefore perform DFT+DMFT calculations for both, the uniform as well as the BD structure (Table I). 
TABLE I. Comparison of the relevant structural parameters in the uniform as well as the bond-disproportionated (111) $2 \mathrm{LNO} / 4 \mathrm{LAO}$ structures optimized in the GGA $+U$ with the experimental structure of bulk $\mathrm{LaNiO}_{3}$ [36]. Atomic coordinates of the uniform and the bond-disproportionated structure are provided in the Appendix.

\begin{tabular}{rrrr}
\hline \hline structure & $\langle\mathrm{Ni}-\mathrm{O}\rangle, \AA$ & $V_{\mathrm{NiO}_{6}}, \AA^{3}$ & $\measuredangle_{\mathrm{Ni}-\mathrm{O}-\mathrm{Ni}}{ }^{\circ}$ \\
\hline uniform & 1.938 & 9.70 & 166.05 \\
$\mathrm{BD}$ & $1.952 / 1.925$ & $9.91 / 9.52$ & 166.04 \\
bulk $\mathrm{LaNiO}_{3}(298 \mathrm{~K})$ & 1.935 & 9.65 & 165.22 \\
\hline \hline
\end{tabular}

\section{B. DFT+DMFT}

Subsequently, self-consistent DFT calculations for both optimized structures (uniform and BD) were performed using WIEN2K [37]. Both GGA band structures (not shown) feature a well-separated manifold crossing the Fermi level, the respective bands are formed by the antibonding combination of $\mathrm{Ni} e_{g}$ and $\mathrm{O} p$ states. Since we have two Ni atoms per cell (see Fig. 1, a, d) and two $e_{g}$ orbitals per $\mathrm{Ni}$, the total number of bands in the manifold is four. For these four bands, we construct the maximally localized Wannier functions (WF) using WANNIER90 [38] via the WIEn2WANNIER interface [39]. Finally, we calculate $H(k)$ by Fourier transforming the Wannier Hamiltonian on a $48 \times 48 \times 1 k$-mesh.

DMFT calculations were performed using the continuous-time quantum Monte Carlo (CT-QMC) in the hybridization expansion (CT-HYB) [40] as implemented in W2DYNAMICS [41, 42]. We used the rotationally invariant Kanamori interaction $U^{\prime}=U-2 J$, which, in addition to the density-density interaction, accounts also for the spin flip and pair hopping terms. By fixing the Hund's exchange $J$ to $0.75 \mathrm{eV}$ [43], we varied the inter-orbital Coulomb repulsion $U^{\prime}$ from 2 to $5 \mathrm{eV}$ [44] and scanned the temperature range between 145 and $450 \mathrm{~K}\left(80 \geq \beta \geq 25 \mathrm{eV}^{-1}\right)$. At every DMFT step, two independent two-orbital impurity problems (for each $\mathrm{Ni}$ atom) in the unit cell were solved. State-of-the-art DFT+DMFT calculations involve full charge-self-consistency, which plays an important role in heterostructures with a sizable electron transfer [45]. Here, we employed the non-charge-self-consistent scheme, i.e. the chemical potential was adjusted to have two electrons per unit cell in each DMFT iteration. We however found that the resulting per-atom occupations in DMFT were similar to the GGA ones. The absence of appreciable charge transfer between the Ni sites a posteriori justifies the usage of a non-charge-self-consistent DFT+DMFT (see the discussion in Ref. 46).

The quasiparticle renormalization is estimated from the slope of the imaginary part of the self-energy $\operatorname{Im} \Sigma\left(i \omega_{n}\right)$ which depends on $\omega_{n}$ linearly at low Matsubara frequencies:

$$
Z \simeq\left(1-\frac{\partial \operatorname{Im} \Sigma\left(i \omega_{n}\right)}{\partial \omega_{n}}\right)^{-1}
$$

For each DMFT calculation, we considered only those $\operatorname{Im} \Sigma\left(i \omega_{n}\right)$ that still lie on a straight line according to a $\chi^{2}$ fit.

\section{Spectral functions}

For selected values of $\beta$ and $U$, we present spectral functions. For these we used self-energies $\Sigma(i \omega)$ computed with the worm algorithm [47], and analytically continued them to the real axis using MAXENT [48] which employs the maximum entropy method [49]. The resulting self-energies $\boldsymbol{\Sigma}(\omega)$ are used to calculate the interacting Green's function on the real frequency axis:

$$
\mathbf{G}^{-1}(\vec{k}, \omega)=(\omega+i \delta+\mu) \mathbf{I}-\mathbf{H}(\vec{k})-\boldsymbol{\Sigma}(\omega)-\boldsymbol{\Sigma}_{\mathrm{dc}}
$$

where matrices in terms of orbitals and sites of the cell are denoted bold, $\boldsymbol{\Sigma}_{\mathrm{dc}}$ is the double-counting correction in the fully localized limit [50] and I the identity matrix. The $\vec{k}$-resolved and $\vec{k}$-integrated spectral functions can be obtained as

$$
A(\vec{k}, \omega)=-\frac{1}{\pi}\left(\frac{1}{m}\right) \operatorname{Tr}[\operatorname{Im} \mathbf{G}(\vec{k}, \omega)]
$$

and

$$
A(\omega)=\left(\frac{1}{N_{\vec{k}}}\right) \sum_{\vec{k} \in \mathrm{BZ}} A(\vec{k}, \omega)
$$

respectively, where $m$ is the dimension of the $\mathbf{H}(\vec{k})$ matrix. These spectral functions are based on $H(\vec{k})$ with the periodic boundary conditions along both in-plane directions, i.e. the Hamiltonian is defined on a torus. To address the edge states, we resort to mixed boundary conditions of a cylinder, which is periodic along $x$ and open along $y$ [51]. The respective Hamiltonian $\mathbf{H}\left(k_{x}\right)$ is now a $n_{y} m \times n_{y} m$ matrix, where $n_{y}$ is the number of unit cells along the open direction, and the Green's function is

$$
\mathbf{G}^{-1}\left(k_{x}, \omega\right)=(\omega+i \delta+\mu) \mathbf{I}-\mathbf{H}\left(k_{x}\right)-\boldsymbol{\Sigma}(\omega)-\boldsymbol{\Sigma}_{\mathrm{dc}}
$$

The respective spectral function is

$$
A\left(k_{x}, \omega\right)=-\frac{1}{\pi}\left(\frac{1}{n_{y} m}\right) \operatorname{Tr}\left\{\operatorname{Im}\left[\mathbf{G}\left(k_{x}, \omega\right)\right]\right\}
$$


Since we are primarily interested in the edge states, we also explicitly calculate their contribution to the spectral weight as:

$A^{\text {edge }}\left(k_{x}, \omega\right)=-\frac{1}{\pi}\left(\frac{1}{2 m}\right) \operatorname{Tr}\left\{\operatorname{Im}\left[\mathbf{G}_{\mathrm{TT}}\left(k_{x}, \omega\right)+\mathbf{G}_{\mathrm{BB}}\left(k_{x}, \omega\right)\right]\right.$

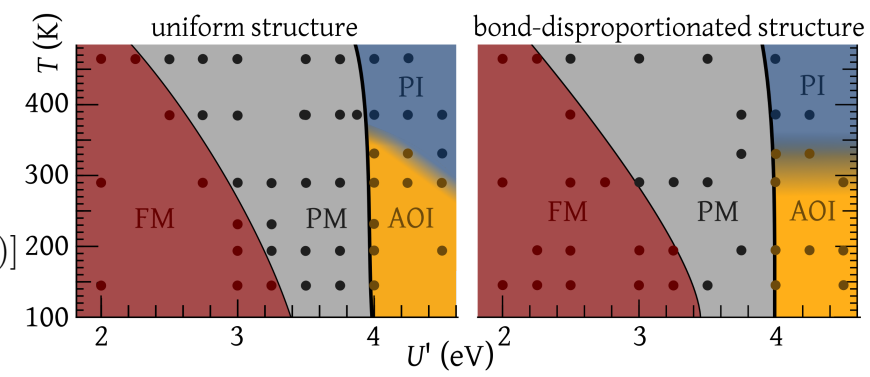

where $\mathbf{G}_{\mathrm{TT}}\left(\mathbf{G}_{\mathrm{BB}}\right)$ denotes the Green's function projected onto the top (bottom) cell.

\section{Choice of the model}

Before turning to the DMFT results, we address a controversially discussed issue of the minimal model for nickelates. The hybridization of $\mathrm{Ni} e_{g}$ states and the $\sigma$-bonded $\mathrm{O} p$ states gives rise to molecular-like $d p_{\sigma}$ orbitals. In a unit cell of $n \mathrm{Ni}$ atoms, the antibonding states form an isolated $\frac{1}{4}$-filled manifold of $2 n$ bands at the Fermi energy. For low-energy excitations, it is seemingly natural to restrict the analysis to these states and use the respective antibonding $d p_{\sigma}$ orbitals as a basis in real space. This minimal two-orbital model, known as the $d$-only model, has been employed in early DMFT studies [20, 52].

On the other hand, the high oxidation state of $\mathrm{Ni}^{3+}$ can lead to a very small, possibly even negative charge transfer gap. In this case, also the low-energy physics will be largely affected by charge transfer processes between $d$ and $p$ states. Indeed, DMFT calculations for such $d+p$ models yielded qualitatively different results [53], mainly because the $e_{g}^{2}$ oxygen ligand hole (L) configuration resulting from the negative charge transfer forms a spin $S=1$ on the Ni sites [54]. It has been further suggested that every second Ni site forms a spin singlet with two ligand holes [55-57], leaving only localized $S=1$ states on the other half of the Ni sites. One should carefully note however that whether one has a negative charge transfer $\left(d^{8}\right)$ or not $\left(d^{7}\right)$ very sensitively depends on the relative position of oxygen and $\mathrm{Ni} e_{g}$ states. In DFT, the oxygen bands are too close to the Fermi level, which would favor the negative charge transfer $d^{8} L$ picture. On top of this, the DFT+DMFT double counting and possible inclusion of the $d-p$ interaction make a theoretical prediction unreliable. Hence, in our view, this question has to be answered by experiment eventually. In this respect, there are indications of a $d^{8} L$ configuration from x-ray absorption spectroscopy for smaller rare earth cations such as $\mathrm{NdNiO}_{3}$ [58], but not for bulk $\mathrm{LaNiO}_{3}$ : very recent single-crystal experiments [59] yield the ordered magnetic moment of $\sim 0.3 \mu_{\mathrm{B}}$, which is far too low for $S=1$.

In fact a $\mathrm{BD}$ scenario can be realized also in a $d$-only model as has been acknowledged long ago [60]. Recent DFT+DMFT calculations by Subedi et al. showed that the BD phase sets in if $\left(U_{d}-3 J_{d}\right)$ is smaller than the difference between the on-site energies of the $e_{g}$ orbitals [61],

FIG. 2. (Color online) DFT+DMFT phase diagram for the uniform structure (left) and the bond disproportionated structure (right). FM, PM, PI, and AOI stand for ferromagnetic metal, paramagnetic metal, paramagnetic metal, and antiferro-orbitally-ordered insulator. Every thick point denotes a separate DMFT calculation.

which in our case is zero (degenerate $e_{g}$ orbitals). This result demonstrates that the emergence of the $\mathrm{BD}$ phase, and hence, the nature of the metal-insulator transition in bulk nickelates are reproduced by a $d$-only model, albeit with a strongly reduced Coulomb interaction $U_{d}$.

In view of this and the unclear experimental situation, we restrict ourselves to the $d$-only model. It features a considerably smaller number of free (and prospectively very sensitive) parameters; and because the effective Coulomb repulsion in the $d$-model can be strongly reduced [61], we scan a broad range of $U_{d}$.

\section{DFT+DMFT RESULTS}

\section{A. Uniform structure}

We start with the uniform structure, for which $\mathrm{DFT}+U$ calculations yield a FM Dirac metal, nearly independent of the $U$ value. Our DMFT $\left(U^{\prime}, T\right)$ phase diagram (Fig. 2, left) reveals a much more involved picture, with four different phases: a ferromagnetic metal (FM) at low $U^{\prime}$, a paramagnetic metal (PM), an antiferroorbitally ordered insulator (AOI), and a paramagnetic insulator (PI); see Fig. 3 and Fig. 4 for the $k$-integrated and $k$-resolved $e_{g}$ spectral functions, respectively. The long-range ferromagnetic ordering transition temperature $T_{\mathrm{C}}$ depends on the onsite Coulomb repulsion: while $U^{\prime} \leq 3 \mathrm{eV}$ yield a ferromagnetic state at room temperature, larger $U^{\prime} \geq 3.5 \mathrm{eV}$ strongly disfavor spin polarization in the studied temperature range. In contrast, the metal-insulator transition (the thick line in Fig. 2) occurs at the critical $U^{\prime}$ which is slightly smaller than $4 \mathrm{eV}$. The nearly vertical line separating PM and insulating phases indicates thermal fluctuations play a minor role in the metal-insulator transition. In the insulating part of the phase diagram, the high-temperature paramagnetic phase (PI) develops an orbital polarization upon cooling, with a gradual crossover to the AOI phase. 


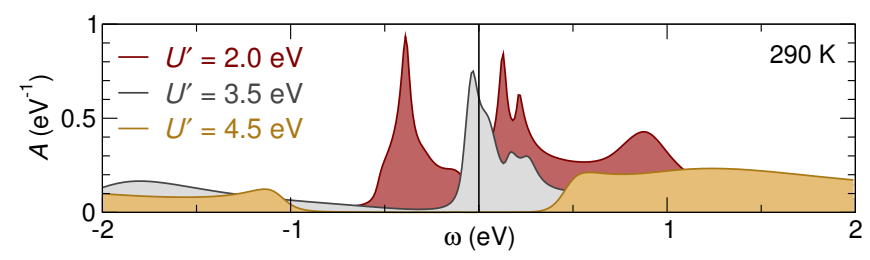

FIG. 3. (Color online) Spectral functions $A(\omega)$ [Eq. (4)] of the uniform structure calculated with DMFT at room temperature $(T=290 \mathrm{~K})$ for the FM phase $\left(U^{\prime}=2 \mathrm{eV}\right)$, the PM phase $\left(U^{\prime}=3.5 \mathrm{eV}\right)$ and the AOI phase $\left(U^{\prime}=4.5 \mathrm{eV}\right)$

a. FM phase The existence of a FM phase is seemingly in agreement with the $\mathrm{DFT}+U$ results that yield a Dirac metal state for the uniform structure. However, the spin-resolved spectral function $A(\omega, k)$ (Fig. 4, top) reveals that our FM phase is not a Dirac metal: the band crossing at the $\mathrm{K}$ point lies $\sim 0.1 \mathrm{eV}$ above the Fermi level (Fig. 4). This effect comes primarily from the real part of the self-energy, which shifts the majority states towards higher frequencies. Instead, the majority states at the Fermi surface form a loop around the K point (Fig. 5, middle).

b. PM phase Above $T_{\mathrm{C}}$, the LNO bilayer is a paramagnetic metal, where both $\mathrm{Ni}$ sites, both orbitals and both spin channels are equally occupied with 0.25 electrons per site and orbital. Correlation effects manifest themselves in the sizable quasiparticle renormalization [Eq. (1)] which amounts to $\sim 0.35-0.60$ depending on the $U^{\prime}$ value. The spectral function (Fig. 4, middle) shows a weakly dispersive feature at the Fermi level, which is largely broadened by the enhanced $\operatorname{Im} \Sigma(0)$. As a result, the Fermi surface plot lacks sharp features (Fig. 5, right).

c. PI and AOI phases Similar to the PM phase, both orbitals and both spin channels are equally populated also in the PI phase, but the spectral function has a gap which grows with $U^{\prime}$. There is an orbital disproportionation between the two $e_{g}$ orbitals setting in at $\sim 350 \mathrm{~K}$, and already at room temperature, a sizable orbital polarization develops. The two neighboring $\mathrm{NiO}_{6}$ octahedra have different predominantly occupied orbitals, giving rise to an antiferro-orbital order (AOI). Interestingly, this spontaneous symmetry breaking occurs despite the degeneracy of the $e_{g}$ orbitals, and hence is of a purely electronic origin. The spectral function (Fig. 4, bottom) shows a wide gap between two incoherent continua - the lower and the upper Hubbard bands.

The degree of the orbital polarization $p$ in nickelates is typically defined $[62,63]$ as

$$
p=\left|\frac{n_{3 z^{2}-r^{2}}-n_{x^{2}-y^{2}}}{n_{3 z^{2}-r^{2}}+n_{x^{2}-y^{2}}}\right|,
$$

where $n_{3 z^{2}-r^{2}}$ and $n_{x^{2}-y^{2}}$ are orbital occupations (a summation over both spin channels is implied). The polarization $p$ is shown in Fig. 6 (left) as a function of temperature for $U^{\prime}=4 \mathrm{eV}$. A sharp increase of orbital
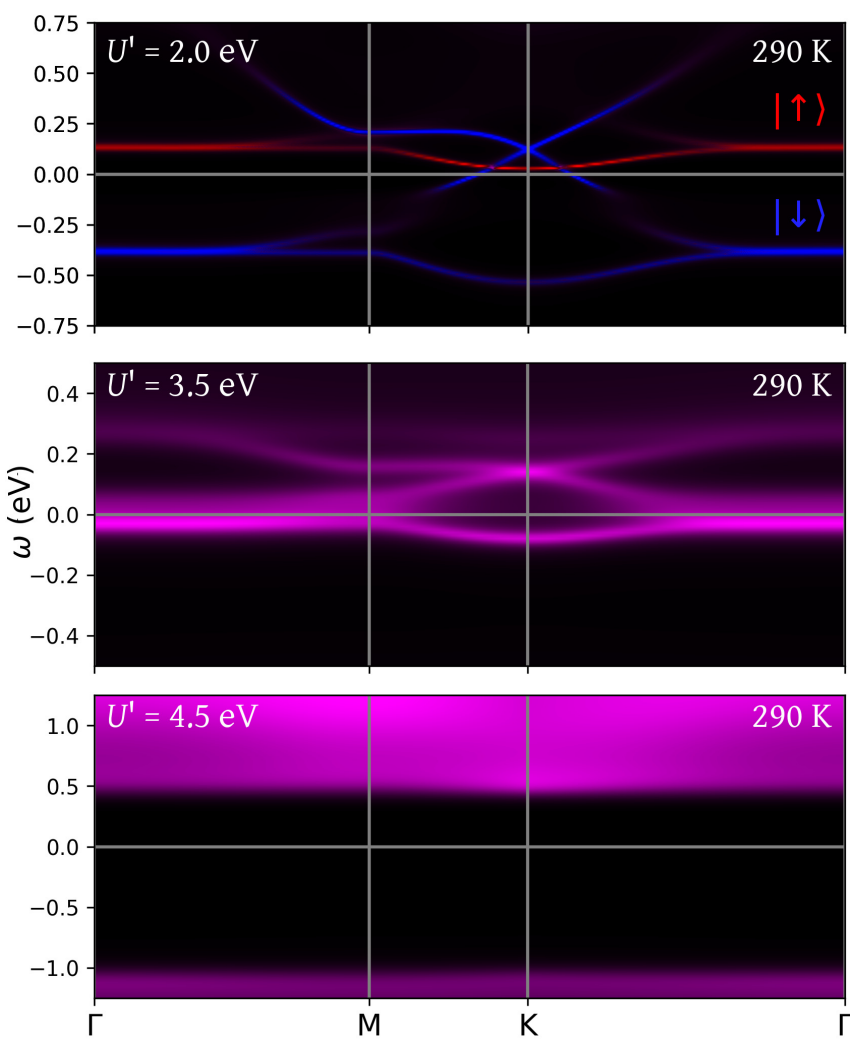

FIG. 4. (Color online) $k$-resolved spectral functions [Eq. (3)] of the uniform structure calculated with DMFT at room temperature $(T=290 \mathrm{~K})$ for the FM phase $\left(U^{\prime}=2 \mathrm{eV}\right)$, the PM phase $\left(U^{\prime}=3.5 \mathrm{eV}\right)$ and the AOI phase $\left(U^{\prime}=4.5 \mathrm{eV}\right)$. In the FM phase, the Dirac point at $\mathrm{K}$ lies $\sim 100 \mathrm{meV}$ above the Fermi level. The two colors correspond to the spectral weight in the majority $(|\downarrow\rangle)$ or minority $(|\uparrow\rangle)$ channel.

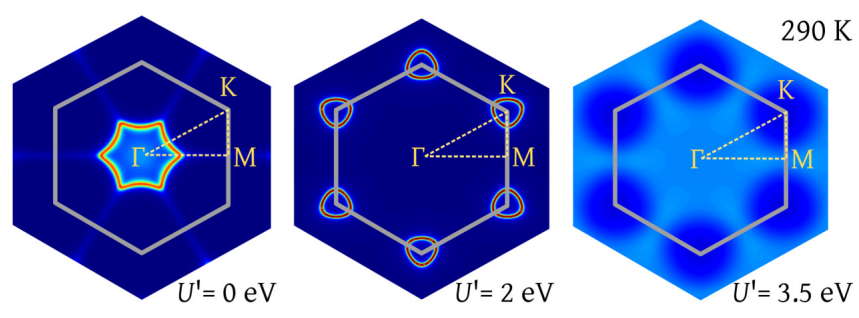

FIG. 5. (Color online) Fermi surfaces of the uniform structure at room temperature. Left: the non-interacting Hamiltonian (DFT). Central: the ferromagnetic metal $\left(U^{\prime}=2 \mathrm{eV}\right)$. Right: the paramagnetic metal $\left(U^{\prime}=3.5 \mathrm{eV}\right)$.

polarization is seen below $\sim 350 \mathrm{~K}$, signaling the phase transition from the PI to the AOI phase.

\section{B. Bond-disproportionated structure}

For the BD structure, $\mathrm{DFT}+U$ calculations with $U$ from a reasonable range yield a semiconductor with a gap of about $0.05 \mathrm{eV}[22]$, in contrast to the Dirac metal state 


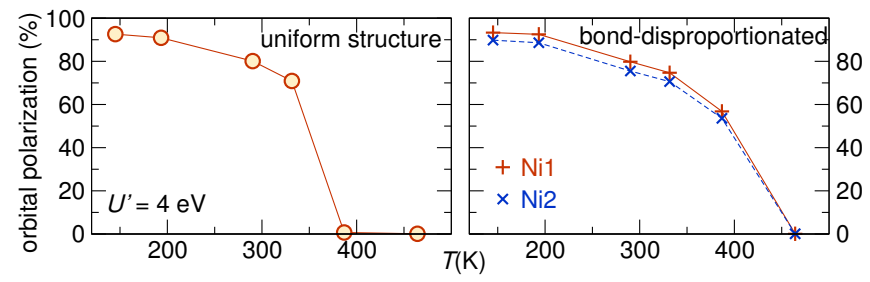

FIG. 6. (Color online) DMFT orbital polarization $p=$ $\left|\left(n_{3 z^{2}-r^{2}}-n_{x^{2}-y^{2}}\right) /\left(n_{3 z^{2}-r^{2}}+n_{x^{2}-y^{2}}\right)\right|$ in the insulating phase $\left(U^{\prime}=4 \mathrm{eV}\right)$ as a function of temperature. The lines are a guide to the eye.

of the uniform structure. Surprisingly, our DFT+DMFT phase diagram for the $\mathrm{BD}$ structure (Fig. 2, right) is very similar to that of the uniform structure. The four emerging phases are analogous to those of the uniform structure, expect for the slight charge disproportionation between the Ni sites that naturally occurs for a BD structure $[53,55]$. Two noticeable differences are i) the shift of boundaries of both phase transitions $(\mathrm{FM} \rightarrow \mathrm{PM}$ and $\mathrm{PM} \rightarrow \mathrm{PI} / \mathrm{AOI}$ ) towards larger $U^{\prime}$ values and ii) the crossover between the AOI and PI phases showing an even weaker dependence on $U^{\prime}$ than in the uniform case. Please note that the charge disproportionation of the starting BD Hamiltonian is very small and hardly affected by the DMFT correlations. Instead, DMFT correlations support again the orbital polarization, see Fig. 6 (right), which is not present in the DFT-derived BD Wannier Hamiltonian.

\section{DISCUSSION}

\section{A. Topological properties}

The honeycomb lattice has an excellent potential for the formation of topological edge states. The emergence of topological states in (111) bilayers of $e_{g}$ electrons has been addressed on the model level $[14,18]$ and in the context of nickelate heterostructures [17, 19, 21, 64]. Hartree-Fock calculations [17-19] yield a rich phase diagram with orbitally ordered and topological phases, but direct $\mathrm{DFT}+U$ calculations favor a conventional ferromagnetic phase [19]. Lattice distortions, and in particular, the breathing distortion can drive the system away from a topological phase [21], as confirmed by direct $\mathrm{DFT}+U$ calculations for LNO bilayers [22]. But in the absence of lattice distortions, $\mathrm{DFT}+U$ yields a Dirac metal state.

In the above studies, with the notable exception of Ref. 64, electronic correlations were either neglected or taken into account at the Hartree-Fock level. DMFT accounts for all local Feynman diagrams, and in this way represents a systematic and substantial improvement over the Hartree-Fock method. On the DMFT level, the electronic correlations are described by the frequencydependent self-energy. In general, the self-energy is a ma- trix with nonzero orbital offdiagonal elements. However, for LNO bilayers, the proximity of the $\mathrm{Ni}-\mathrm{O}-\mathrm{Ni}$ angles (165.81 and $165.58^{\circ}$ in the uniform and the BD structure, respectively) to $180^{\circ}$ leads to vanishingly small offdiagonal elements between $3 z^{2}-r^{2}$ and $x^{2}-y^{2}$ orbitals. As a result, we can safely neglect offdiagonal elements of the hybridization function $F\left(i \omega_{n}\right)$ in our impurity problems, leading to the self-energies $\Sigma\left(i \omega_{n}\right)$ that are diagonal in the site-orbital-spin basis.

The resulting DMFT self-energies are used to calculate the interacting Greens function using Eq. (5) and subsequently, the spectral functions using Eqs. (6) and (7). To this end, we use the DMFT self-energy of our bulk calculation for all sites and consider periodic boundary conditions along the $x$ axis and open boundary conditions for the $y$ axis, leading to the cylinder geometry. The spectral functions for the FM, PM, and AOI states are shown in Fig. 7. Only the FM state shows a distinct edge state, which however lies entirely in the unoccupied part of the spectrum, $\sim 0.1-0.2 \mathrm{eV}$ above the Fermi energy. This agrees with the position of the Dirac point in Fig. 4 (top). Both PM and AOI phases yield very incoherent features, without any distinct edge states. We therefore conclude that the emergence of topological states in (111) LNO bilayers is unlikely.

\section{B. Comparison with model DMFT calculations}

By performing DFT + DMFT calculations in the $d$ basis, we actually solve a two-site two-orbital Hubbard model at quarter filling $(n=1)$. The phase diagram of this model based on a simplified (typically, semi-elliptic) density of states has been studied in the literature, in particular within DMFT, and it is tempting to put our DFT + DMFT results into this context.

The early Hirsch-Fye QMC results showed a remarkable stabilization of ferromagnetism in a two-orbital Hubbard model away from half-filling due to the Hund's coupling [65]. A pronounced tendency towards orbital ordering for the quarter filling has been found in the same study [65]. The interplay between magnetic and orbital ordering has been further addressed by Peters et al. [66, 67], who employed numerical renormalization group (NRG) to solve auxiliary impurity problems. For quarter-filling at zero temperature, they found a first-order metal-insulator transition between the FM state and the ferromagnetic counterpart of our AOI state, driven by the increased interorbital interaction $U^{\prime}$ [66]. Looking at our DFT+DMFT phase diagrams, one may speculate that the FM metallic phase extends to large $U^{\prime}$ values at lower temperatures than accessible by CTQMC and shown in Fig. 2. Further, the AOI order actually supports an insulating ferromagnetic phase through superexchange. Hence one may speculate further that at lower temperatures, an additional ferromagnetic ordering occurs in the AOI phase. This would yield two close-by ferromagnetic phases, similar as was discussed 

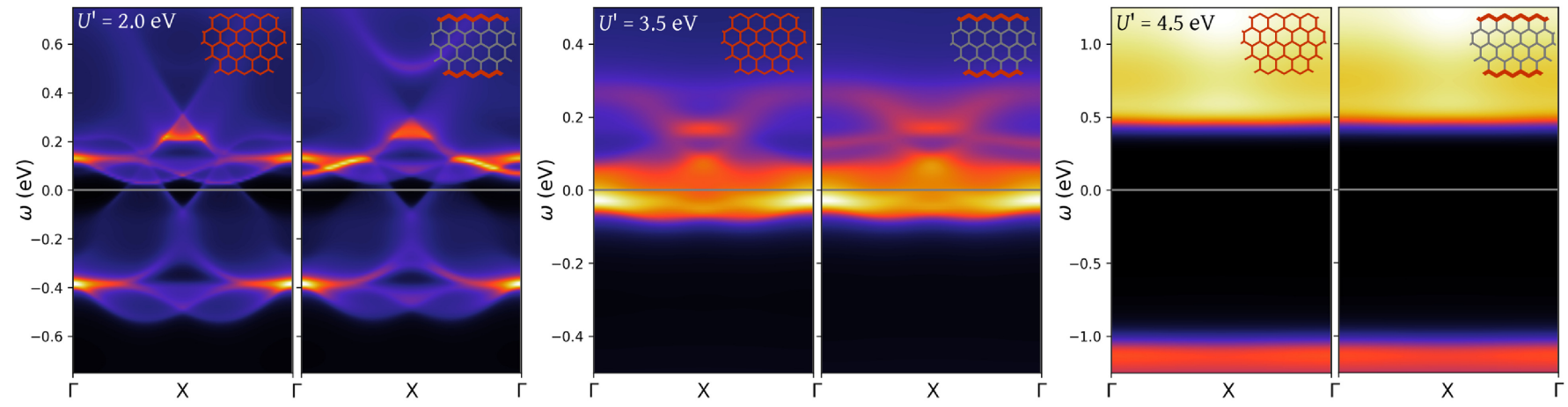

FIG. 7. (Color online) DFT+DMFT $A\left(k_{x}, \omega\right)$ at room temperature on cylinders of 50 unit cells. In each panel, the left spectrum shows the total $A\left(k_{x}, \omega\right)$ [Eq. (6)], while the right spectrum shows the weight of the edge states $A^{\text {edge }}\left(k_{x}, \omega\right)[$ Eq. $(7)]$, as schematically depicted in the top-right corners of each plot. Note the topological edge state visible in the edge-projected (right) spectrum lying 0.1-0.2 eV above the Fermi energy for the $U^{\prime}=2.0 \mathrm{eV}$ (left panel).

in Ref. 66 for a two-orbital model. In this case, a huge magnetoresistance can be expected [66].

A subsequent paper reports a detailed model study of the quarter-filled case [67]. The ground state phase diagram features, in addition to the two ferromagnetic and a paramagnetic phase, also the AOI phase, albeit in a narrow region of the phase diagram, where the interorbital repulsion $U^{\prime}$ largely exceeds the Hund's exchange. One of the main results of Ref. 67 is the stabilization of the orbital order without Jahn-Teller distortions. Our DFT+DMFT calculations not only corroborate this conclusion, but strengthen it further: the presence of the AOI phase in the BD structure implies that the antiferroorbital order is resilient to the competing mechanism of a breathing distortion.

\section{Comparison with experiments}

Recent transport measurements on $2 \mathrm{LNO} / 4 \mathrm{LAO}$ heterostructures yield a band gap of $120 \mathrm{meV}$ [24], which grows if the thickness of the LAO layer increases, which has been argued to stem from the accumulation of defects in thicker layers. Nevertheless, the insulating nature of the LNO bilayers can be regarded as a sound experimental result as it also concurs with the earlier report [23]. Thus, we infer that the inter-orbital repulsion $U^{\prime}$ in LNO bilayers exceeds $4 \mathrm{eV}$, as only such high values yield an insulating phase (Fig. 2).

At the first glance, we do not have arguments in favor or against the breathing distortion: the insulating part of the phase diagram is similar for both structures, and the $\mathrm{DFT}+U$ energies are essentially degenerate. But the bare emergence of the AOI phase already indicates a tendency towards a cooperative Jahn-Teller distortion (orbital ordering). Hence, in the insulating state, the electronic degrees of freedom disfavor the competing mechanism of a breathing distortion even in the BD structure. The presence of such an orbital order can be verified experimentally by measuring x-ray linear dichroism (see Ref. 68), because both the uniform and the BD structure show a very strong orbital polarization $p$ already at room temperature (Fig. 6). Nickelate heterostructures with a sizable orbital polarization do exist for the (001) case [68]. In contrast to the (001) case, the AOI state in (111) LNO bilayers is an insulating state, and a large orbital polarization is easier to achieve. In several cases, the MottHubbard metal-insulator transition is indeed accompanied by an orbital polarization, e.g. in $\mathrm{V}_{2} \mathrm{O}_{3}$ [69] and $\mathrm{SrVO}_{3}$ films [70].

Magnetic properties of LNO bilayers remain hitherto unexplored, but the recent study of $\mathrm{NdNiO}_{3}$ bilayers on LAO, reporting antiferromagnetic correlations and orbital order [71], demonstrates that an experimental insight is feasible. According to our DFT+DMFT results, the insulating phases, PI and AOI, do not show any magnetic order above $\sim 150 \mathrm{~K}$, and it would be interesting to verify this result experimentally.

A good agreement between our DFT + DMFT and model DMFT studies (Sec. IV B) gives hope that the low-temperature physics of (111) LNO bilayers can be even more exciting. In particular, a very high magnetoresistance was found at the boundary between the antiferro-orbitally-ordered (insulating) and the orbitallydisordered (metallic) ferromagnetic phases in the model DMFT [67]. Although we do not see the former phase in the phase diagram (Fig. 2), it may become stabilized by cooling. Unfortunately, performing CT-QMC at low temperature becomes prohibitively challenging, although very recent developments such as the superstate sampling method [72] can largely alleviate the computational effort. Nonetheless our phase diagram sets the stage of what orders can be expected in experiment.

\section{SUMMARY AND OUTLOOK}

Using DFT+DMFT calculations, we evaluated the phase diagram of a (111) oxide heterostructure formed by $\mathrm{LaNiO}_{3}$ bilayers interleaved with four layers of $\mathrm{LaAlO}_{3}$, 
in a wide range of temperatures and the values of the inter-orbital Coulomb repulsion $U^{\prime}$. Independent of the presence or absence of breathing distortions that are typical for bulk nickelates, we find four phases: a ferromagnetic and a paramagnetic metal, a paramagnetic insulator, as well as an antiferro-orbitally-ordered insulator. Spectral functions calculated on cylinders feature edge states in the ferromagnetic metallic state, whereas both insulating phases are topologically trivial. Taking the experimentally observed activated behavior as an indication for a insulating state, we argue that $\mathrm{LaNiO}_{3}$ bilayers can develop sizable orbital polarization at room temperature. Based on earlier model DMFT studies, we can expect ferromagnetic ordering at lower temperatures, offering an intriguing possibility of a transition between a metallic and insulating ferromagnetic phases, with a concomitant high magnetoresistance.

Compared to DFT $+U$, DFT + DMFT provides a more realistic treatment of electronic correlations and gives access to finite temperature properties. However, DMFT is restricted to local correlations. For a quasi-2D system with the low coordination number such as nickelate (111) bilayers, nonlocal correlation effects can play an important role. A natural extension of our study would be the application of cluster $[73,74]$ or diagrammatic [75] extensions of DMFT to the phase diagram of $2 \mathrm{LNO} / 4 \mathrm{LAO}$.

\section{ACKNOWLEDGMENTS}

We acknowledge financial support by European Research Council under the European Union's Seventh Framework Program (FP/2007-2013)/ERC through grant agreement n. 306447. OJ was supported by the Austrian Science Fund (FWF) through the Lise Meitner programme, project no. M2050. We thank Marta Gibert, Sumanta Bhandary, and Gang Li for fruitful discussions. Calculations have been done on the Vienna Scientific Cluster (VSC).
[1] M. Imada, A. Fujimori, and Y. Tokura, Metal-insulator transitions, Rev. Mod. Phys. 70, 1039 (1998).

[2] S.-W. Cheong and M. Mostovoy, Multiferroics: a magnetic twist for ferroelectricity, Nature Mater. 6, 13 (2007).

[3] Y. Tokura and S. Seki, Multiferroics with spiral spin orders, Adv. Mater. 22, 1554 (2010).

[4] Y. Tokura, S. Seki, and N. Nagaosa, Multiferroics of spin origin, Rep. Prog. Phys. 77, 076501 (2014).

[5] A. P. Ramirez, Colossal magnetoresistance, J. Phys.: Condens. Matter 9, 8171 (1997).

[6] Y. Tokura, Critical features of colossal magnetoresistive manganites, Rep. Rrog. Phys. 69, 797 (2006).

[7] W. E. Pickett, Electronic structure of the hightemperature oxide superconductors, Rev. Mod. Phys. 61, 433 (1989).

[8] P. A. Lee, N. Nagaosa, and X.-G. Wen, Doping a Mott insulator: Physics of high-temperature superconductivity, Rev. Mod. Phys. 78, 17 (2006).

[9] A. Ohtomo and H. Y. Hwang, A high-mobility electron gas at the $\mathrm{LaAlO}_{3} / \mathrm{SrTiO}_{3}$ heterointerface, Nature (London) 427, 423 (2004).

[10] A. Bhattacharya, S. J. May, S. G. E. te Velthuis, M. Warusawithana, X. Zhai, B. Jiang, J.-M. Zuo, M. R. Fitzsimmons, S. D. Bader, and J. N. Eckstein, Metalinsulator transition and its relation to magnetic structure in $\left.\left(\mathrm{LaMnO}_{3}\right)_{2 n} / \mathrm{SrMnO}_{3}\right)_{n}$ superlattices, Phys. Rev. Lett. 100, 257203 (2008).

[11] E. Assmann, P. Blaha, R. Laskowski, K. Held, S. Okamoto, and G. Sangiovanni, Oxide heterostructures for efficient solar cells, Phys. Rev. Lett. 110, 078701 (2013).

[12] L. Wang, Y. Li, A. Bera, C. Ma, F. Jin, K. Yuan, W. Yin, A. David, W. Chen, W. Wu, W. Prellier, S. Wei, and T. Wu, Device performance of the Mott insulator $\mathrm{LaVO}_{3}$ as a photovoltaic material, Phys. Rev. Applied 3, 064015 (2015).

[13] A. Ohtomo, D. A. Muller, J. L. Grazul, and H. Y.
Hwang, Artificial charge-modulation in atomic-scale perovskite titanate superlattices, Nature (London) 419, 378 (2002).

[14] D. Xiao, W. Zhu, Y. Ran, N. Nagaosa, and S. Okamoto, Interface engineering of quantum Hall effects in digital transition metal oxide heterostructures, Nature Commun. 2, 596 (2011).

[15] F. D. M. Haldane, Model for a quantum Hall effect without Landau levels: Condensed-matter realization of the "parity anomaly", Phys. Rev. Lett. 61, 2015 (1988).

[16] L. Si, O. Janson, G. Li, Z. Zhong, Z. Liao, G. Koster, and K. Held, Quantum anomalous Hall state in ferromagnetic $\mathrm{SrRuO}_{3}$ (111) bilayers, Phys. Rev. Lett. 119, 026402 (2017).

[17] K.-Y. Yang, W. Zhu, D. Xiao, S. Okamoto, Z. Wang, and Y. Ran, Possible interaction-driven topological phases in (111) bilayers of $\mathrm{LaNiO}_{3}$, Phys. Rev. B 84, 201104 (2011).

[18] A. Rüegg and G. A. Fiete, Topological insulators from complex orbital order in transition-metal oxides heterostructures, Phys. Rev. B 84, 201103 (2011).

[19] A. Rüegg, C. Mitra, A. A. Demkov, and G. A. Fiete, Electronic structure of $\left(\mathrm{LaNiO}_{3}\right)_{2} /\left(\mathrm{LaAlO}_{3}\right)_{N}$ heterostructures grown along [111], Phys. Rev. B 85, 245131 (2012).

[20] P. Hansmann, X. Yang, A. Toschi, G. Khaliullin, O. K. Andersen, and K. Held, Turning a nickelate Fermi surface into a cupratelike one through heterostructuring, Phys. Rev. Lett. 103, 016401 (2009).

[21] A. Rüegg, C. Mitra, A. A. Demkov, and G. A. Fiete, Lattice distortion effects on topological phases in $\left(\mathrm{LaNiO}_{3}\right)_{2} /\left(\mathrm{LaAlO}_{3}\right)_{N}$ heterostructures grown along the [111] direction, Phys. Rev. B 88, 115146 (2013).

[22] D. Doennig, W. E. Pickett, and R. Pentcheva, Confinement-driven transitions between topological and Mott phases in $\left(\mathrm{LaNiO}_{3}\right)_{N} /\left(\mathrm{LaAlO}_{3}\right)_{M}$ (111) superlattices, Phys. Rev. B 89, 121110 (2014).

[23] S. Middey, D. Meyers, M. Kareev, E. J. Moon, B. A. 
Gray, X. Liu, J. W. Freeland, and J. Chakhalian, Epitaxial growth of (111)-oriented $\mathrm{LaAlO}_{3} / \mathrm{LaNiO}_{3}$ ultra-thin superlattices, Appl. Phys. Lett. 101, 261602 (2012).

[24] H. Wei, M. Grundmann, and M. Lorenz, Confinementdriven metal-insulator transition and polarity-controlled conductivity of epitaxial $\mathrm{LaNiO}_{3} / \mathrm{LaAlO}_{3}$ (111) superlattices, Appl. Phys. Lett. 109, 082108 (2016).

[25] V. I. Anisimov, A. I. Poteryaev, M. A. Korotin, A. O. Anokhin, and G. Kotliar, First-principles calculations of the electronic structure and spectra of strongly correlated systems: dynamical mean-field theory, J. Phys.: Condens. Matter 9, 7359 (1997).

[26] A. I. Lichtenstein and M. I. Katsnelson, Ab initio calculations of quasiparticle band structure in correlated systems: LDA++ approach, Phys. Rev. B 57, 6884 (1998).

[27] G. Kotliar, S. Y. Savrasov, K. Haule, V. S. Oudovenko, O. Parcollet, and C. A. Marianetti, Electronic structure calculations with dynamical mean-field theory, Rev. Mod. Phys. 78, 865 (2006).

[28] K. Held, Electronic structure calculations using dynamical mean field theory, Adv. Phys. 56, 829 (2007).

[29] O. Janson, Z. Zhong, G. Sangiovanni, and K. Held, Dynamical mean field theory for oxide heterostructures, in Spectroscopy of Complex Oxide Interfaces, Springer Series in Materials Science, Vol. 266, edited by C. Cancellieri and V. Strocov (Springer International Publishing, 2018) pp. 215-243, arXiv:1611.09044.

[30] A. I. Liechtenstein, V. I. Anisimov, and J. Zaanen, Density-functional theory and strong interactions: Orbital ordering in Mott-Hubbard insulators, Phys. Rev. B 52, R5467 (1995).

[31] J. A. Alonso, J. L. García-Muñoz, M. T. Fernández-Díaz, M. A. G. Aranda, M. J. Martínez-Lope, and M. T. Casais, Charge disproportionation in $R \mathrm{NiO}_{3}$ perovskites: Simultaneous metal-insulator and structural transition in $\mathrm{YNiO}_{3}$, Phys. Rev. Lett. 82, 3871 (1999).

[32] M. Medarde, M. T. Fernández-Díaz, and P. Lacorre, Long-range charge order in the low-temperature insulating phase of $\mathrm{PrNiO}_{3}$, Phys. Rev. B 78, 212101 (2008).

[33] J. L. García-Muñoz, M. A. G. Aranda, J. A. Alonso, and M. J. Martínez-Lope, Structure and charge order in the antiferromagnetic band-insulating phase of $\mathrm{NdNiO}_{3}$, Phys. Rev. B 79, 134432 (2009).

[34] A. Hampel and C. Ederer, Interplay between breathing mode distortion and magnetic order in rare-earth nickelates $R \mathrm{NiO}_{3}$ within DFT $+U$, Phys. Rev. B 96, 165130 (2017).

[35] G. Kresse and J. Furthmüller, Efficient iterative schemes for $a b$ initio total-energy calculations using a plane-wave basis set, Phys. Rev. B 54, 11169 (1996); Efficiency of ab-initio total energy calculations for metals and semiconductors using a plane-wave basis set, Comput. Mater. Sci. 6, 15 (1996).

[36] J. L. García-Muñoz, J. Rodríguez-Carvajal, P. Lacorre, and J. B. Torrance, Neutron-diffraction study of $\mathrm{RNiO}_{3}$ ( $R=\mathrm{La}, \mathrm{Pr}, \mathrm{Nd}, \mathrm{Sm})$ : Electronically induced structural changes across the metal-insulator transition, Phys. Rev. B 46, 4414 (1992).

[37] K. Schwarz, P. Blaha, and G. Madsen, Electronic structure calculations of solids using the WIEN2k package for material sciences, Comp. Phys. Commun. 147, 71 (2002).

[38] A. A. Mostofi, J. R. Yates, Y.-S. Lee, I. Souza, D. Vanderbilt, and N. Marzari, wannier90: A tool for obtaining maximally-localised Wannier functions, Comp. Phys.
Commun. 178, 685 (2008)

[39] J. Kuneš, R. Arita, P. Wissgott, A. Toschi, H. Ikeda, and K. Held, Wien2wannier: From linearized augmented plane waves to maximally localized Wannier functions, Comput. Phys. Commun. 181, 1888 (2010).

[40] P. Werner, A. Comanac, L. de' Medici, M. Troyer, and A. J. Millis, Continuous-time solver for quantum impurity models, Phys. Rev. Lett. 97, 076405 (2006).

[41] N. Parragh, A. Toschi, K. Held, and G. Sangiovanni, Conserved quantities of $S U(2)$-invariant interactions for correlated fermions and the advantages for quantum Monte Carlo simulations, Phys. Rev. B 86, 155158 (2012).

[42] M. Wallerberger, A. Hausoel, P. Gunacker, A. Kowalski, N. Parragh, F. Goth, K. Held, and G. Sangiovanni, w2dynamics: Local one- and two-particle quantities from dynamical mean field theory, arXiv:1801.10209.

[43] Note that $\mathrm{SU}(2)$ symmetric interactions require a smaller $J$ compared to the Slater parametrization used in $\mathrm{DFT}+U: U_{\text {Kanamori }}=U_{\text {Slater }}+\frac{8}{7} J_{\text {Slater }}$ and $J_{\text {Kanamori }}=$ $\frac{5}{7} J_{\text {Slater }}$. See Supplementary Note 1 in [76] for details.

[44] The $U_{d}$ value in DMFT calculation is considerably smaller than $U_{d}$ in $\mathrm{DFT}+U$ due to the different spatial extent of the orbitals to which they are applied: DMFT operates on Wannier orbitals, having substantial Ni and $\mathrm{O}$ contributions, while in $\mathrm{DFT}+U$, repulsion pertains to the spatially confined atomic $d$-orbital of $\mathrm{Ni}$.

[45] F. Lechermann, L. Boehnke, and D. Grieger, Formation of orbital-selective electron states in $\mathrm{LaTiO}_{3} / \mathrm{SrTiO}_{3}$ superlattices, Phys. Rev. B 87, 241101 (2013); F. Lechermann and M. Obermeyer, Towards Mott design by $\delta$ doping of strongly correlated titanates, New J. Phys. 17, 043026 (2015).

[46] S. Bhandary, E. Assmann, M. Aichhorn, and K. Held, Charge self-consistency in density functional theory combined with dynamical mean field theory: $k$-space reoccupation and orbital order, Phys. Rev. B 94, 155131 (2016).

[47] P. Gunacker, M. Wallerberger, E. Gull, A. Hausoel, G. Sangiovanni, and K. Held, Continuous-time quantum Monte Carlo using worm sampling, Phys. Rev. B 92, 155102 (2015).

[48] R. Levy, J. P. F. LeBlanc, and E. Gull, Implementation of the maximum entropy method for analytic continuation, Comput. Phys. Commun. 215, 149 (2017).

[49] M. Jarrell and J. E. Gubernatis, Bayesian inference and the analytic continuation of imaginary-time quantum Monte Carlo data, Phys. Rep. 269, 133 (1996).

[50] V. I. Anisimov, I. V. Solovyev, M. A. Korotin, M. T. Czyžyk, and G. A. Sawatzky, Density-functional theory and $\mathrm{NiO}$ photoemission spectra, Phys. Rev. B 48, 16929 (1993).

[51] We note that the honeycomb lattice allows for two inequivalent terminations: the zigzag edge and the armchair edge. All calculations in this study are performed for the zigzag edge.

[52] P. Hansmann, A. Toschi, X. Yang, O. K. Andersen, and K. Held, Electronic structure of nickelates: From twodimensional heterostructures to three-dimensional bulk materials, Phys. Rev. B 82, 235123 (2010).

[53] M. J. Han, X. Wang, C. A. Marianetti, and A. J. Millis, Dynamical mean-field theory of nickelate superlattices, Phys. Rev. Lett. 107, 206804 (2011).

[54] N. Parragh, G. Sangiovanni, P. Hansmann, S. Hummel, 
K. Held, and A. Toschi, Effective crystal field and Fermi surface topology: A comparison of $d$ - and $d p$-orbital models, Phys. Rev. B 88, 195116 (2013).

[55] H. Park, A. J. Millis, and C. A. Marianetti, Site-selective Mott transition in rare-earth-element nickelates, Phys. Rev. Lett. 109, 156402 (2012).

[56] R. J. Green, M. W. Haverkort, and G. A. Sawatzky, Bond disproportionation and dynamical charge fluctuations in the perovskite rare-earth nickelates, Phys. Rev. B 94, 195127 (2016).

[57] K. Haule and G. L. Pascut, Mott transition and magnetism in rare earth nickelates and its fingerprint on the X-ray scattering, Sci. Rep. 7, 10375 (2017).

[58] V. Bisogni, S. Catalano, R. J. Green, M. Gibert, R. Scherwitzl, Y. Huang, V. N. Strocov, P. Zubko, S. Balandeh, J.-M. Triscone, G. Sawatzky, and T. Schmitt, Ground-state oxygen holes and the metal-insulator transition in the negative charge-transfer rare-earth nickelates, Nat. Commun. 7, 13017 (2016).

[59] H. Guo, Z. Li, L. Zhao, Z. Hu, C. Chang, C.-Y. Kuo, W. Schmidt, A. Piovano, O. Sobolev, D. Khomskii, L. Tjeng, and A. Komarek, Antiferromagnetic correlations in the metallic strongly correlated transition metal oxide $\mathrm{LaNiO}_{3}$, Nat. Commun. 9, 43 (2018).

[60] I. I. Mazin, D. I. Khomskii, R. Lengsdorf, J. A. Alonso, W. G. Marshall, R. M. Ibberson, A. Podlesnyak, M. J. Martínez-Lope, and M. M. Abd-Elmeguid, Charge ordering as alternative to Jahn-Teller distortion, Phys. Rev. Lett. 98, 176406 (2007).

[61] A. Subedi, O. E. Peil, and A. Georges, Low-energy description of the metal-insulator transition in the rareearth nickelates, Phys. Rev. B 91, 075128 (2015).

[62] M. Wu, E. Benckiser, M. W. Haverkort, A. Frano, Y. Lu, U. Nwankwo, S. Brück, P. Audehm, E. Goering, S. Macke, V. Hinkov, P. Wochner, G. Christiani, S. Heinze, G. Logvenov, H.-U. Habermeier, and B. Keimer, Strain and composition dependence of orbital polarization in nickel oxide superlattices, Phys. Rev. B 88, 125124 (2013).

[63] H. Park, A. J. Millis, and C. A. Marianetti, Influence of quantum confinement and strain on orbital polarization of four-layer $\mathrm{LaNiO}_{3}$ superlattices: A DFT+DMFT study, Phys. Rev. B 93, 235109 (2016).

[64] S. Okamoto, W. Zhu, Y. Nomura, R. Arita, D. Xiao, and N. Nagaosa, Correlation effects in (111) bilayers of perovskite transition-metal oxides, Phys. Rev. B 89, 195121 (2014).

[65] K. Held and D. Vollhardt, Microscopic conditions favor- ing itinerant ferromagnetism: Hund's rule coupling and orbital degeneracy, Eur. Phys. J. B 5, 473 (1998).

[66] R. Peters and T. Pruschke, Orbital and magnetic order in the two-orbital Hubbard model, Phys. Rev. B 81, 035112 (2010).

[67] R. Peters, N. Kawakami, and T. Pruschke, Orbital order, metal-insulator transition, and magnetoresistance effect in the two-orbital Hubbard model, Phys. Rev. B 83, 125110 (2011).

[68] A. S. Disa, D. P. Kumah, A. Malashevich, H. Chen, D. A. Arena, E. D. Specht, S. Ismail-Beigi, F. J. Walker, and C. H. Ahn, Orbital engineering in symmetry-breaking polar heterostructures, Phys. Rev. Lett. 114, 026801 (2015).

[69] G. Keller, K. Held, V. Eyert, D. Vollhardt, and V. I. Anisimov, Electronic structure of paramagnetic $\mathrm{V}_{2} \mathrm{O}_{3}$ : Strongly correlated metallic and Mott insulating phase, Phys. Rev. B 70, 205116 (2004).

[70] Z. Zhong, M. Wallerberger, J. M. Tomczak, C. Taranto, N. Parragh, A. Toschi, G. Sangiovanni, and K. Held, Electronics with correlated oxides: $\mathrm{SrVO}_{3} / \mathrm{SrTiO}_{3}$ as a Mott transistor, Phys. Rev. Lett. 114, 246401 (2015).

[71] S. Middey, D. Meyers, D. Doennig, M. Kareev, X. Liu, Y. Cao, Z. Yang, J. Shi, L. Gu, P. J. Ryan, R. Pentcheva, J. W. Freeland, and J. Chakhalian, Mott electrons in an artificial graphenelike crystal of rare-earth nickelate, Phys. Rev. Lett. 116, 056801 (2016).

[72] A. Kowalski, A. Hausoel, P. Gunacker, and G. Sangiovanni, State- and superstate-sampling in hybridizationexpansion continuous-time quantum Monte Carlo, arXiv:1807.00361.

[73] A. I. Lichtenstein and M. I. Katsnelson, Antiferromagnetism and $d$-wave superconductivity in cuprates: A cluster dynamical mean-field theory, Phys. Rev. B 62, R9283 (2000).

[74] G. Kotliar, S. Y. Savrasov, G. Pálsson, and G. Biroli, Cellular dynamical mean field approach to strongly correlated systems, Phys. Rev. Lett. 87, 186401 (2001).

[75] G. Rohringer, H. Hafermann, A. Toschi, A. A. Katanin, A. E. Antipov, M. I. Katsnelson, A. I. Lichtenstein, A. N. Rubtsov, and K. Held, Diagrammatic routes to nonlocal correlations beyond dynamical mean field theory, Rev. Mod. Phys. 90, 025003 (2018).

[76] A. Hausoel, M. Karolak, E. Şaşıŏ̆lu, A. Lichtenstein, K. Held, A. Katanin, A. Toschi, and G. Sangiovanni, Local magnetic moments in iron and nickel at ambient and Earth's core conditions, Nature Commun. 8, 16062 (2017). 


\section{Appendix: DFT $+U$-optimized crystal structures of 2LNO/4LAO (111) heterostructures}

Crystal structures used for DFT+DMFT calculations are provided in Table II.

TABLE II. Atomic coordinates of the uniform and the bond-disproportionated structures used for DFT+DMFT calculations. Space group P3 (143), $a=5.3646 \AA$, $c=13.30 \AA$.

\begin{tabular}{|c|c|c|c|c|c|c|c|}
\hline \multirow{2}{*}{ atom } & \multirow{2}{*}{ Wyckoff position } & \multicolumn{3}{|c|}{ uniform structure } & \multicolumn{3}{|c|}{ bond disproportionated } \\
\hline & & $x / a$ & $y / b$ & $z / c$ & $x / a$ & $y / b$ & $z / c$ \\
\hline $\mathrm{Ni}$ & $1 b$ & $\frac{1}{3}$ & $\frac{2}{3}$ & -0.08557 & $\frac{1}{3}$ & $\frac{2}{3}$ & -0.08477 \\
\hline $\mathrm{Ni}$ & $1 c$ & $\frac{2}{3}$ & $\frac{1}{3}$ & 0.08557 & $\frac{2}{3}$ & $\frac{1}{3}$ & 0.08678 \\
\hline $\mathrm{Al}$ & $1 a$ & 0 & 0 & 0.25335 & 0 & 0 & 0.25466 \\
\hline $\mathrm{Al}$ & $1 b$ & $\frac{1}{3}$ & $\frac{2}{3}$ & 0.41745 & $\frac{1}{3}$ & $\frac{2}{3}$ & 0.41828 \\
\hline $\mathrm{Al}$ & $1 c$ & $\frac{2}{3}$ & $\frac{1}{3}$ & 0.58255 & $\frac{2}{3}$ & $\frac{1}{3}$ & 0.58333 \\
\hline $\mathrm{Al}$ & $1 a$ & 0 & 0 & 0.74665 & 0 & 0 & 0.74754 \\
\hline $\mathrm{La}$ & $1 a$ & 0 & 0 & 0.00000 & 0 & 0 & 0.00000 \\
\hline $\mathrm{La}$ & $1 b$ & $\frac{1}{3}$ & $\frac{2}{3}$ & 0.16501 & $\frac{1}{3}$ & $\frac{2}{3}$ & 0.16584 \\
\hline $\mathrm{La}$ & $1 c$ & $\frac{2}{3}$ & $\frac{1}{3}$ & 0.33234 & $\frac{2}{3}$ & $\frac{1}{3}$ & 0.33393 \\
\hline $\mathrm{La}$ & $1 a$ & 0 & 0 & 0.50000 & 0 & 0 & 0.50145 \\
\hline $\mathrm{La}$ & $1 b$ & $\frac{1}{3}$ & $\frac{2}{3}$ & 0.66766 & $\frac{1}{3}$ & $\frac{2}{3}$ & 0.66908 \\
\hline $\mathrm{La}$ & $1 c$ & $\frac{2}{3}$ & $\frac{1}{3}$ & 0.83499 & $\frac{2}{3}$ & $\frac{1}{3}$ & 0.83563 \\
\hline $\mathrm{O}$ & $3 d$ & 0.00001 & 0.45619 & 0.00000 & 0.45754 & 0.00284 & 0.00222 \\
\hline $\mathrm{O}$ & $3 d$ & 0.32833 & 0.20833 & 0.16906 & 0.32947 & 0.20851 & 0.17008 \\
\hline $\mathrm{O}$ & $3 d$ & 0.12891 & 0.33333 & 0.33440 & 0.12916 & 0.33333 & 0.33495 \\
\hline $\mathrm{O}$ & $3 d$ & 0.00000 & 0.53725 & 0.50000 & 0.00000 & 0.53765 & 0.50040 \\
\hline $\mathrm{O}$ & $3 d$ & 0.33333 & 0.12892 & 0.66560 & 0.33333 & 0.12845 & 0.66596 \\
\hline $\mathrm{O}$ & $3 d$ & 0.20833 & 0.32833 & 0.83094 & 0.20853 & 0.32728 & 0.83139 \\
\hline
\end{tabular}

